\title{
Herzinfarkt durch Weichmacher in Plastik?
}

\begin{abstract}
Bisphenol A (BPA) wird für Beschichtungen, Verpackungsmaterial, Haushaltsutensilien und vieles mehr verwendet. Der Stoff macht Plastik weich und ermöglicht damit erst die Verarbeitung dieses Materials. Es häufen sich Hinweise, dass die Chemikalie gesundheitsschädlich ist. Höhere Urin-BPA-Konzentrationen (uBPA) sind in Querschnittsuntersuchungen unabhängig von den traditionellen Risikofaktoren mit Herzerkrankungen assoziiert.
\end{abstract}

- Eine aktuelle Studie von Wissenschaftlern der University of Exeter in Exeter (UK) zeigte einen Zusammenhang mit Herzerkrankungen. In einer Querschnittsstudie mit mehr als 1500 Probanden über 10,8 Jahre konnte nachgewiesen werden, dass Personen mit höheren BPA-Mengen im Urin überdurch- schnittlich häufig an Angina pectoris und Herzinfarkten erkrankten.

\section{- D. Melzer et al.}

Urinary bisphenol: A concentration and risk of future coronary artery disease in apparently healthy men and women. Published online before print February 21, 2012, doi: 10.1161/ CIRCULATIONAHA.111.069153

\section{Kommentar}

Die Autoren warnen vor voreiligen Schlüssen. Die Studie weist nur auf den statistischen Zusammenhang zwischen BPA und Herzerkrankungen hin, sie beweist nicht, dass die Chemikalie der Verursacher ist. Weitere Untersuchungen sind dazu notwendig. Sollte sich in diesen der Verdacht bestätigen, dann wäre das BPA allerdings neben Rauchen, Hypertonie und erhöhtem Blutcholesterin als zusätzlicher Herzrisikofaktor zu beachten.

K. MALBERG =

\section{Die besten Programme zur Gewichtsabnahme}

\section{Übergewicht ist ein dominierendes Gesundheitsproblem in unserer heutigen Gesellschaft. Nachdem kürzlich gezeigt werden konnte, dass das kommerzielle Programm "Weight Watchers" bei der Gewichstreduktion zu besseren Ein- Jahres-Ergebnissen führt als ein Hausarztberatungsprogramm, wurden nun mehrere Programme miteinander verglichen.}

- Aus 17 Praxen in England wurden 587 Patienten mit einem BMI $\geq 30 \mathrm{~kg} / \mathrm{m}^{2}$ rekrutiert und für die Dauer von drei Monaten einem von sieben Armen zugeteilt: Weight Watchers, Slimming World, Rosemary Conley, einem Programm des Nationalen Health Systems (NHS), einem Hausarzt- und einem

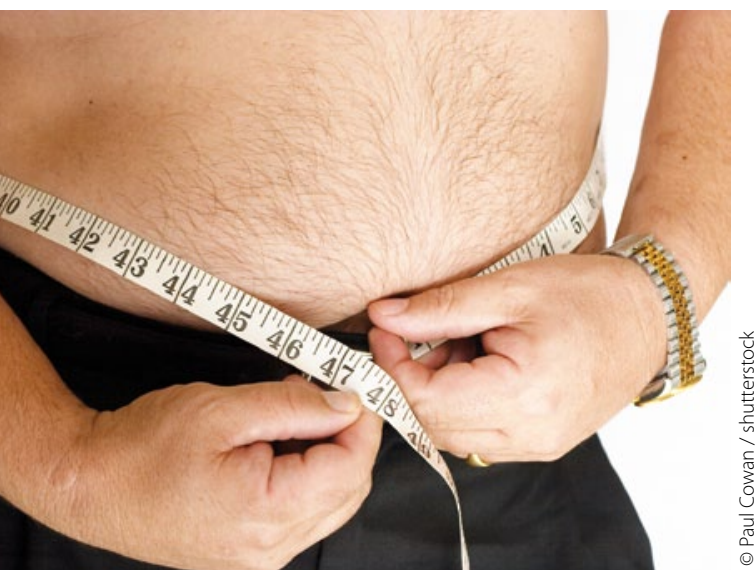

Was können kommerzielle Gewichtswächter, was der Arzt nicht kann?
Apotheken-Programm. Eine weitere Patientengruppe durfte unter diesen Möglichkeiten frei wählen.

Erfreulich ist zunächst einmal, dass die Patienten aller acht Gruppen nach drei Monaten mit dem Ende der Intervention eine im Vergleich zum Ausgangsgewicht signifikante, allerdings deutlich unterschiedliche Gewichtsabnahmen erreichten. Bemerkenswerterweise waren aber nur die Ergebnisse der drei kommerziellen Programme (Weight Watchers, Rosemary Conley und Slimming World) im Vergleich zu den Kontrollen (Fitness-Gruppe) signifikant. Das Gewicht nach einem Jahr war mit Ausnahme der Praxis- und Apothekengruppe gegenüber der Vergleichsgruppe weiterhin erniedrigt. Im Gruppenvergleich war nur Weight Watchers signifikant besser als die Kontrollgruppe. Die von den Autoren errechneten Gesamtkosten lagen für die kommerziellen Programme niedriger als die anderen Methoden.

\section{Kommentar}

Ein kommerzielles Programm zur Gewichtsabnahme (Weight Watchers), das die Teilnehmer normalerweise selbst bezahlen müssen, ist erfolgreicher ist als die Intervention in der Praxis, deren Kosten die Krankenversicherungen übernehmen. Für die Überlegenheit der kommerziellen Programme werden verschiedene Ursachen diskutiert: größere Erfahrung und Motivation der Mitarbeiter, Gruppeneffekte statt individueller Betreuung und günstigere Termine mit regelmäßigerer Teilnahmerate. Untersuchungen zeigen, dass bei den Mitarbeitern in der Primärversorgung der Glaube an Erfolge in der Gewichtsabnahme durch schlechte Erfahrungen verloren gegangen ist. Bei den professionellen Programmen dagegen sindaus welchen Gründen auch immer - Motivation und Engagement ausgeprägt. Entweder müssen also die traditionellen Methoden zur Gewichtsabnahme in der Praxis verbessert werden, oder die Kassen müssen die Kosten für kommerzielle Programme erstatten.

H. HolzGREVE =

- K. Jolly et al.

Comparison of range of commercial or primary care led weight reduction programmes with minimal intervention control for weight loss in obesity: Lighten Up randomised controlled trial. Brit. Med. J. 343 (2011) d6500 